\title{
Supplementary Information for Unexpected Low Mechanical Stability of Titin I27 Domain at Physiologically Relevant Temperature
}

\author{
Miao Yu $\stackrel{\$}{\uparrow}$ J Jung-Hsuan Lu $\stackrel{\$}{\$}$ Shimin Le, ${ }^{*, \ddagger}$ and Jie Yan ${ }^{*,+, \dagger}$ \\ $\dagger$ Mechanobiology Institute, National University of Singapore, Singapore 117411 \\ $\ddagger$ Department of Physics, National University of Singapore, Singapore 117542 \\ E-mail: phyles@nus.edu.sg; phyyj@nus.edu.sg
}

\section{Supplementary Texts}

Supplementary Text S1. Plasmids construct and protein expression.

A plasmid (named as pET151-Avi-8xI27-Spy) was prepared for expressing the protein construct for single-molecule stretching experiments. The DNA fragment containing the coding sequencing of eight repeat of I27 (8xI27) was synthesized by geneArt, and then assembled into a pET151-Avi-(Insert)-Spy plasmid ${ }^{1}$ by HiFi DNA Assembly (NEB). The plasmid was co-transformed with a BirA plasmid and expressed in Escherichia coli BL21 (DE3) cultured in LB-media with D-Biotin (Sigma Aldrich), and affinity purified through 6His-tag. The detailed sequence of the construct is listed below, where the sequences in orange colour are the eight repeats of the 89 amino acids of 27 domain, the sequences in blue and cyan colours

\footnotetext{
$\S$ The first two authors have equal contribution.
} 
are the AviTag and SpyTag, respectively, and the sequences in grey colour are short flexible linkers between neighbouring domains.

MHHHHHHGLNDIFEAQKIEWHEGFEIDKVWYDLDAKLGDIEFIKVNKGGGSGLE LIEVEKPLYGVEVFVGETAHFEIELSEPDVHGQWKLKGQPLAASPDAEIIEDGKKHIL ILHNAQLGMTGEVSFQAANTKSAANLKVKELGGGSGLIEVEKPLYGVEVFVGETAH FEIELSEPDVHGQWKLKGQPLAASPDAEIIEDGKKHILILHNAQLGMTGEVSFQAAN TKSAANLKVKELGGGSGLIEVEKPLYGVEVFVGETAHFEIELSEPDVHGQWKLKGQ PLAASPDAEIIEDGKKHILILHNAQLGMTGEVSFQAANTKSAANLKVKELGGGSGLIE VEKPLYGVEVFVGETAHFEIELSEPDVHGQWKLKGQPLAASPDAEIIEDGKKHILIL HNAQLGMTGEVSFQAANTKSAANLKVKELGGGSGLIEVEKPLYGVEVFVGETAHF EIELSEPDVHGQWKLKGQPLAASPDAEIIEDGKKHILILHNAQLGMTGEVSFQAANT KSAANLKVKELGGGSGLIEVEKPLYGVEVFVGETAHFEIELSEPDVHGQWKLKGQP LAASPDAEIIEDGKKHILILHNAQLGMTGEVSFQAANTKSAANLKVKELGGGSGLIEV EKPLYGVEVFVGETAHFEIELSEPDVHGQWKLKGQPLAASPDAEIIEDGKKHILILH NAQLGMTGEVSFQAANTKSAANLKVKELGGGSGLIEVEKPLYGVEVFVGETAHFEI ELSEPDVHGQWKLKGQPLAASPDAEIIEDGKKHILILHNAQLGMTGEVSFQAANTKS AANLKVKELGSGGGSGAHIVMVDAYKPTK*

\section{Supplementary Text S2. Pseudo dwell time analysis and bootstrap analysis of the force-dependent lifetime of I27 domain.}

During experiments, at each force $F$ and each temperature $T$, the time taken $\tau_{\mathrm{u}, \mathrm{n}}$ from one unfolding event to the next was recorded, where the subscript $n=1, \cdots, 8$ denotes the number of $I 27$ repeats that remain folded prior to the transition. The so-called pseudo dwell time of one I27 domain was calculated as $\tau_{\mathrm{u}}=n \times \tau_{\mathrm{u}, \mathrm{n}} \cdot{ }^{2}$ A schematic illustration is shown in Figure S3.

A bootstrap analysis was then performed on the experimentally obtained data pool of $\tau_{\mathrm{u}}$. Briefly, at each force and each temperature, a new data pool of $\tau_{\mathrm{u}}^{\text {boot }}$ with $\mathrm{M}=100$ data 
points, where each data point was randomly picked from the original experimentally obtained $\tau_{\mathrm{u}}$, was generated. The cumulative distribution of the new data pool of $\tau_{\mathrm{u}}^{\text {boot }}$ was then fitted with an exponential equation $p\left(\tau_{\mathrm{u}}\right)=1-e^{-k_{\mathrm{u}}(F, T) \tau_{\mathrm{u}}}$. Based on the fitting, a value of the unfolding rate of $\mathrm{I} 27, k_{\mathrm{u}}(F, T)$, was obtained. Repeating such procedure for $N_{\mathrm{r}}=10$ times, 10 values of the $k_{\mathrm{u}}(F, T)$ were obtained, from which the mean value, standard deviations, standard errors of $k_{\mathrm{u}}(F, T)$ were therefore calculated.

Repeating such analysis for each force and each temperature, the force- and temperature dependent $k_{\mathrm{u}}(F, T)$ were then obtained (Figure 2 in the main text). Here we note that, the data of force-dependent unfolding rate of 127 at $23{ }^{\circ} \mathrm{C}$ were taken from our previously published paper $^{3}$ for comparison with the data obtained at higher temperatures .

\section{Supplementary Text S3. Temperature- and force-dependent I27 unfolding rate analysis by an Arrhenius law based asymptotic an- alytical expression.}

For rigid folded structures, its force-dependent unfolding rate can be described by an asymp-

totic analytical expression based on Arrhenius Law: $k_{\mathrm{u}}(T, F)=k_{\mathrm{u}, 0}^{\sim}(T) \exp ^{\beta\left(\sigma F-\eta F^{1 / 2}\right)}{ }^{4}$ The two key fitting parameters are $\sigma=L^{*}+b^{*}-b_{0}$ and $\eta=L^{*} \sqrt{\frac{k_{\mathrm{B}} T}{A}}$, where $L^{*}$ is the contour length of the peptide released until reaching the transition state. $b_{0}$ and $b^{*}$ are the linear lengths between the two force-attaching points on the folded cores in the native state and the transition state structures, respectively. $A$ is the bending persistence length of the peptide chain of I27, and has been determined to be $\sim 0.4-0.8 \mathrm{~nm}$ at forces below $100 \mathrm{pN}^{5-7}$ and a value of $0.5 \mathrm{~nm}$ is used in this study for the $A$ of the first strand of peptide chain of $\mathrm{I} 27 .{ }^{8}$ The $k_{\mathrm{u}, 0}^{\sim}(T)$ is a fitting parameter that is related to the zero-force unfolding rates of I27. ${ }^{4}$

For the unfolding of I27 domain in this study where the force was applied to the two termini of I27, denoting $N^{*}$ the corresponding number of the residues in the released peptide, which may be different at different temperatures. The value of $L^{*}$ is then determined by 
$L^{*}=N^{*} \times l_{0}$, where $l_{0}=0.38 \mathrm{~nm}$ is the contour length per residue. ${ }^{9}$ The value of $b_{0}\left(N^{*}\right)$ can be directly measured from the structure (PDB:1tit) ${ }^{10}$ (supplementary Table S1). Hence, the fitting parameters of the above asymptotic analytical expression become $N^{*}$ and $b^{*}$. Since $N^{*}$ is the corresponding number of residues in the released peptide, we can assume it maybe an integers within 15 . Thus, by fitting the experimental measured $k_{\mathrm{u}}(T, F)$ data with the asymptotic analytical expression, the best-fitting parameters $N^{*}$ and $b^{*}$ at different temperatures are indicated in Figure 2.

\section{Supplementary Text S4. The force extension curves of I27 at its folded state, transition state, and unfolded state.}

The force-extension curve of a folded I27 domain are determined by the rigid rotation fluctuation of a characteristic rigid-body with a length $b_{0} \sim 3.2 \mathrm{~nm}$ based on the structure (PDB: 1tit), which is the distance between the two force-attaching points of the rigid body. The force-extension curves can be described by the freely-jointed chain (FJC) polymer model with a single segment: $x_{0}(F)=b_{0}\left(\operatorname{coth}\left(\frac{F b_{0}}{k_{\mathrm{B}} T}\right)-\frac{k_{\mathrm{B}} T}{F b_{0}}\right)$.

Assuming the unfolded I27 exists as a fully unstructured flexible peptide chain, the forceextension curves of the unfolded I27 can be described by the worm-like chain (WLC) polymer

model through the Marko-Siggia formula: $\frac{F A}{k_{\mathrm{B}} T}=\frac{1}{4\left(1-\frac{x_{1}(F)}{l}\right)^{2}}-\frac{1}{4}+\frac{x_{1}(F)}{l}$, where $A \sim 0.4-0.8$ $\mathrm{nm}$ is the bending persistence length of the peptide chain, ${ }^{5-7} l=N \times l_{0}$ is the contour length of the unfolded chain, $N=89$ a.a. is the number of residues of $\mathrm{I} 27, l_{0}=0.38 \mathrm{~nm}$ is the contour length of per residue. ${ }^{9}$ A value of $A=0.8 \mathrm{~nm}$, which is suitable to describe the low force force-extension curve of the unfolded I27 peptide, ${ }^{11}$ was used in this study for the unfolded I27 peptide.

MD simulation studies have proposed that the unfolding of I27 primarily follows a transition pathway of peeling the N-terminal strand (the A- $\mathrm{A}^{\prime}$ peptide), during which the bonded residues in the $\mathrm{A}-\mathrm{A}^{\prime}$ peptide are sequentially peeled off from the remaining folded core until reaching the transition state, where a number $N^{*}$ residue has been peeled off. ${ }^{12-14}$ Hence, the 
force-extension curve of the transition state of I27, $x^{*}(F)$, can be considered as the sum of the force-extension of the peeled unstructured flexible peptide chain and the remaining folded core. The former can be described by WLC model as: $\frac{F A}{k_{\mathrm{B}} T}=\frac{1}{4\left(1-\frac{x_{\text {peeled }}^{*}(F)}{l^{*}}\right)^{2}}-\frac{1}{4}+\frac{x_{\text {peeled }}^{*}(F)}{l^{*}}$, where the $l^{*}=N^{*} \times l_{0}$, and the later can be described by FJC model as $x_{\text {folded }}^{*}(F)=$ $b^{*}\left(\operatorname{coth}\left(\frac{F b^{*}}{k_{\mathrm{B}} T}\right)-\frac{k_{\mathrm{B}} T}{F b^{*}}\right)$. The switching force $F_{\mathrm{s}}$ of the force-dependent unfolding rate is the force point at which the extension of transition state equals that of the folded state. Hence the $F_{\mathrm{s}}$ can be solved by the equation $\delta^{*}\left(F_{\mathrm{s}}\right)=x^{*}\left(F_{\mathrm{s}}\right)-x_{0}\left(F_{\mathrm{s}}\right)=0$ (Figure S6).

Supplementary Table S1: The parameters $\left(N^{*}, b_{0}\left(N^{*}\right)\right.$ and $\left.L^{*}\right)$ measured based on I27 structure (PDB:1tit).

\begin{tabular}{|c|c|c|c|}
\hline $\begin{array}{c}i \text { residue } \\
\text { PDB: } 1 \text { tit }\end{array}$ & $\begin{array}{c}N^{*} \\
\text { to } 15^{\text {th }} \mathrm{V}\end{array}$ & $\begin{array}{c}b_{0}\left(N^{*}\right) \\
i \text { to } 88^{\text {th }} \mathrm{E}\end{array}$ & $\begin{array}{c}L^{*}=l_{0} \times N^{*} \\
l_{0}=0.38 \mathrm{~nm}\end{array}$ \\
\hline $1(\mathrm{~L})$ & 15 & 4.04 & 5.70 \\
\hline $2(\mathrm{I})$ & 14 & 3.79 & 5.32 \\
\hline $3(\mathrm{E})$ & 13 & 3.52 & 4.94 \\
\hline $4(\mathrm{~V})$ & 12 & 3.20 & 4.56 \\
\hline $\mathbf{5}(\mathbf{E})$ & $\mathbf{1 1}$ & $\mathbf{3 . 3 0}$ & $\mathbf{4 . 1 8}$ \\
\hline $6(\mathrm{~K})$ & 10 & 2.94 & 3.80 \\
\hline $\mathbf{7}(\mathrm{P})$ & $\mathbf{9}$ & $\mathbf{2 . 6 6}$ & $\mathbf{3 . 4 2}$ \\
\hline $8(\mathrm{~L})$ & 8 & 2.29 & 3.04 \\
\hline $9(\mathrm{Y})$ & 7 & 2.06 & 2.66 \\
\hline $10(\mathrm{G})$ & 6 & 1.70 & 2.28 \\
\hline $11(\mathrm{~V})$ & 5 & 1.44 & 1.90 \\
\hline $12(\mathrm{E})$ & 4 & 1.07 & 1.52 \\
\hline
\end{tabular}




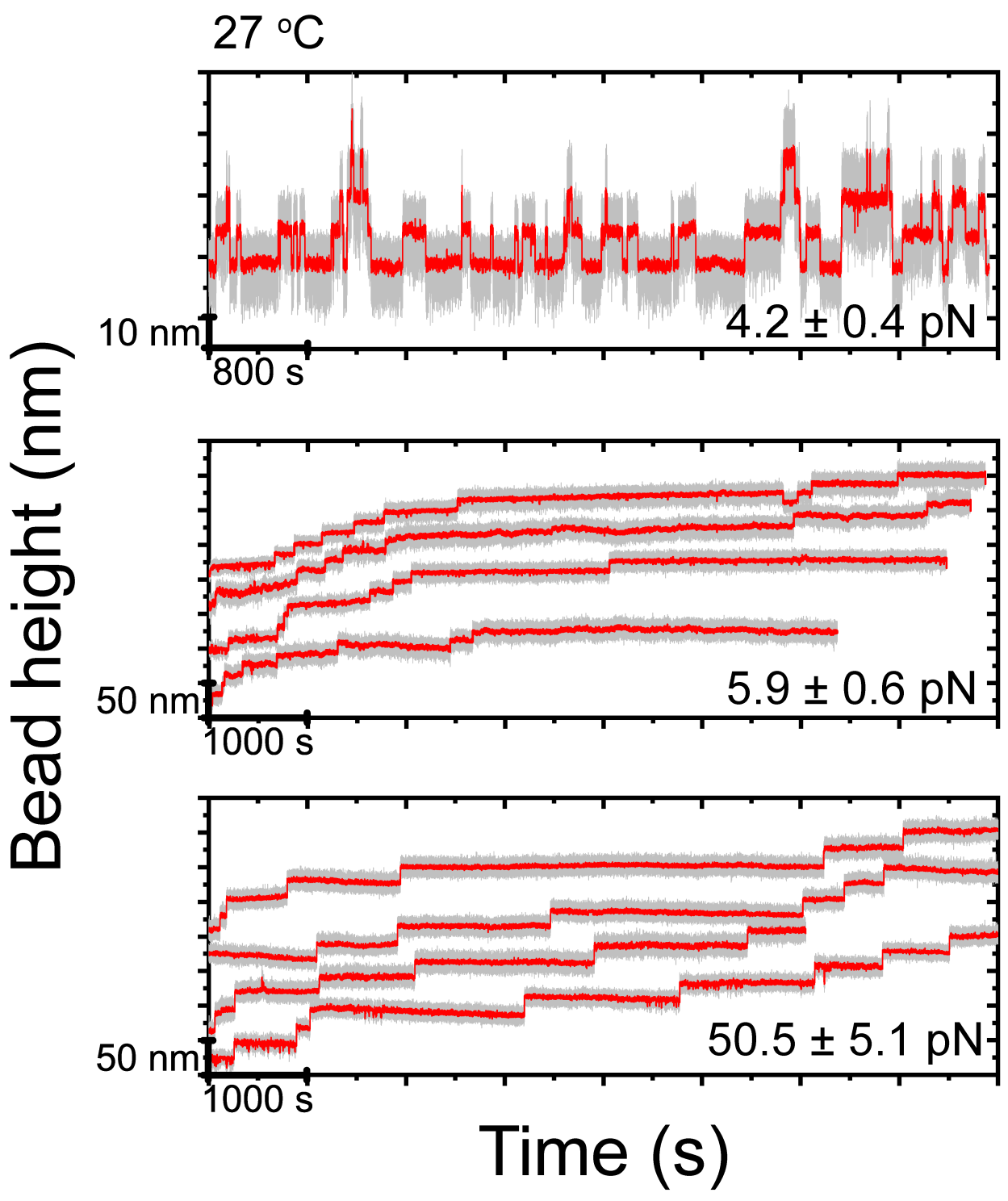

Supplementary Figure S1: Example extension time traces of the I27 unfolding/refolding dynamics at various forces at $27^{\circ} \mathrm{C}$. Representative time traces of the unfolding/refolding events of the construct containing eight repeats of I27 domains at constant forces at $27^{\circ} \mathrm{C}$. The red lines show a FFT-smooth of the raw data (gray). Each stepwise bead height increase indicates an unfolding event of a folded I27, and each stepwise bead height decrease indicates a refolding event of an unfolded I27. 


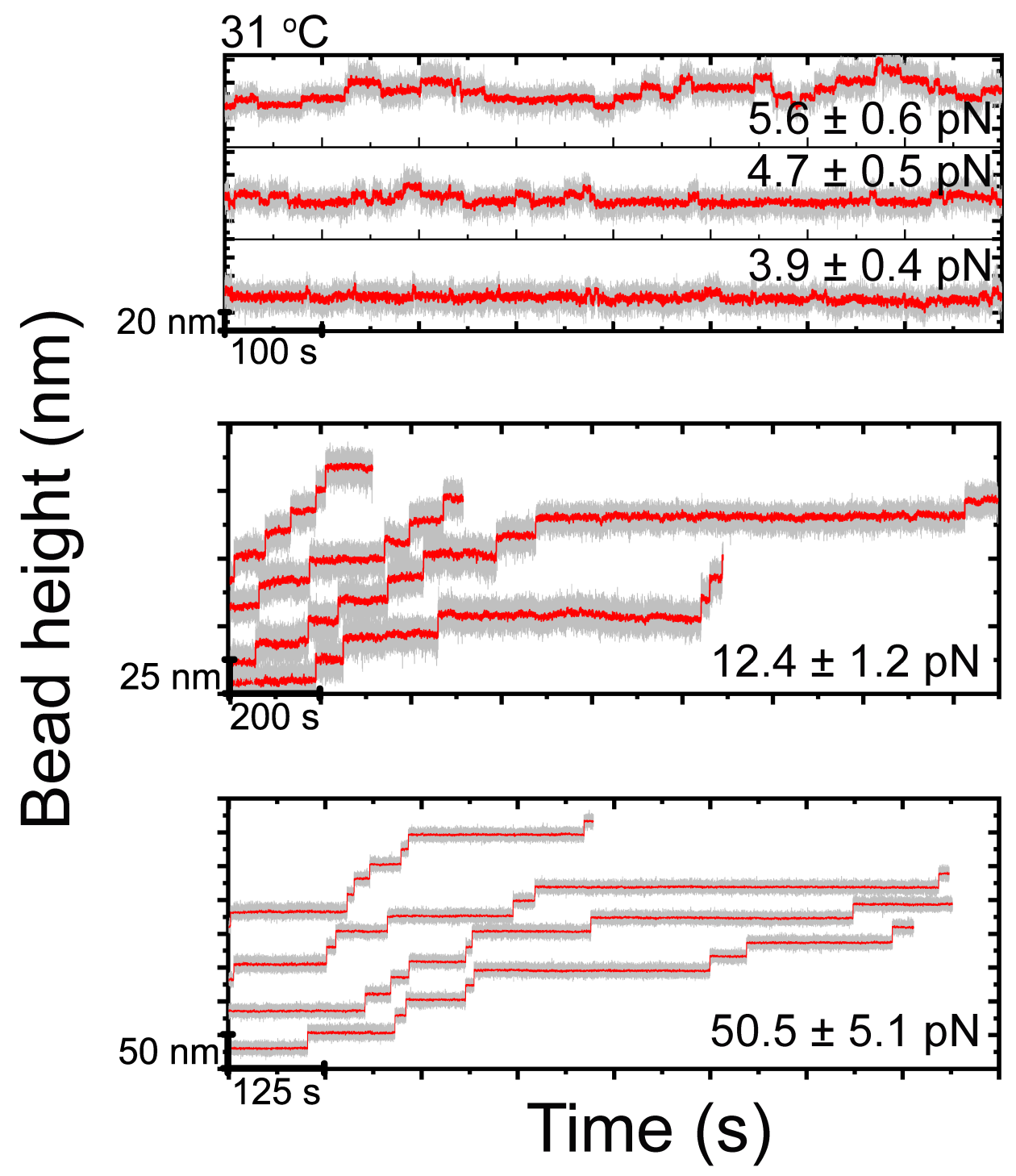

Supplementary Figure S2: Example extension time traces of the I27 unfolding/refolding dynamics at various forces at $31{ }^{\circ} \mathrm{C}$. Representative time traces of the unfolding/refolding events of the construct containing eight repeats of I27 domains at constant forces at $31{ }^{\circ} \mathrm{C}$. The red lines show a FFT-smooth of the raw data (gray). Each stepwise bead height increase indicates an unfolding event of a folded I27, and each stepwise bead height decrease indicates a refolding event of an unfolded I27. 


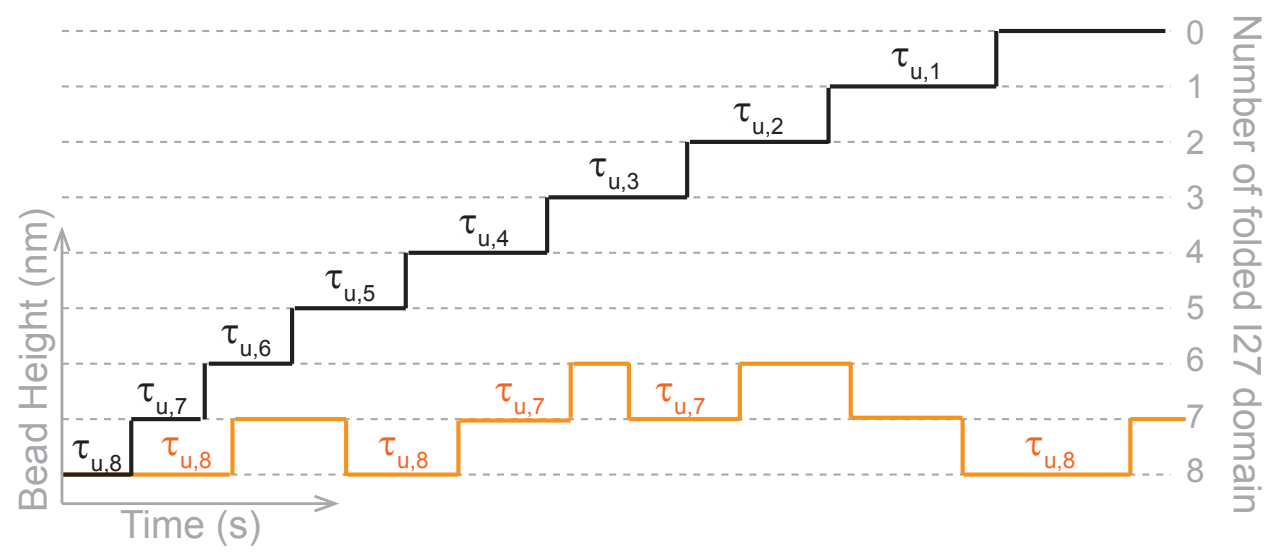

Supplementary Figure S3: Illustration of the lifetime analysis for I27 domain unfolding.. Two example bead height time traces (black and orange) are illustrated on the panel. The pseudo-lifetime of each I27 unfolding event, denoted as $\tau_{\mathrm{u}, \mathrm{n}}$ is indicated, where the $\mathrm{n}$ indicates the number of folded I27 domains before the transition. The so-called pseudo dwell time of one 127 domain was calculated as $\tau_{\mathrm{u}}=n \times \tau_{\mathrm{u}, \mathrm{n}}$ (more details can be found in $^{2}$ ). 

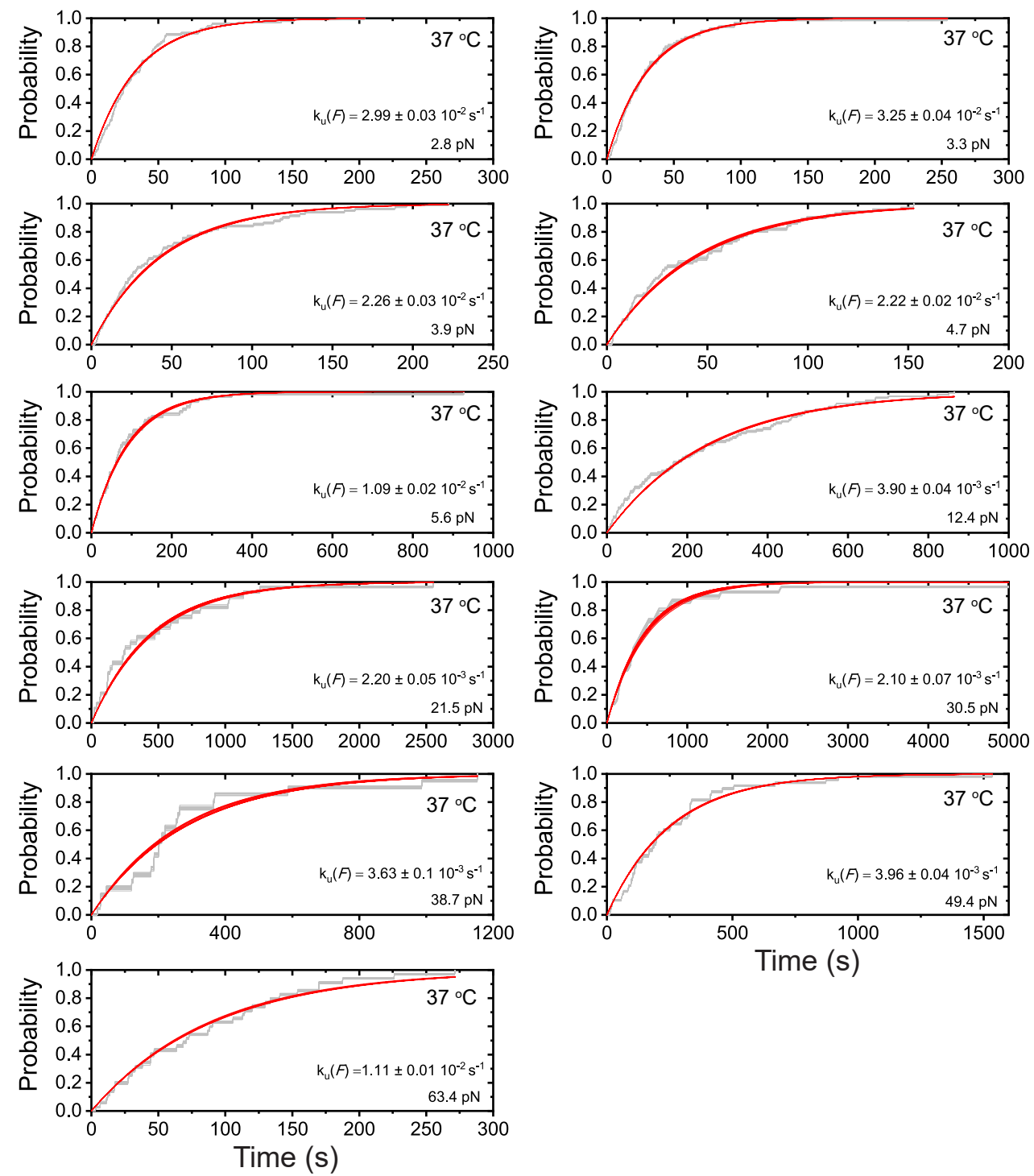

Supplementary Figure S4: The time dependent unfolding probability of I27.. Example time dependent unfolding probability of $\mathrm{I} 27$ at $37^{\circ} \mathrm{C}$. The gray lines show the experimentally obtained time dependent unfolding probability based on bootstrap analysis on the pseudo dwell times. The red lines show the fitting curves to an exponential equation $p_{\mathrm{F}, \mathrm{T}}\left(\tau_{u}\right)=1-e^{-k_{u}(F, T) \tau_{u}}$. The corresponding force and fitted $k_{u}(F, T)$ are indicated in the panel. The bootstrap analysis and the corresponding fitting are repeated 10 times to obtain the standard error of the $k_{u}(F, T)$. 


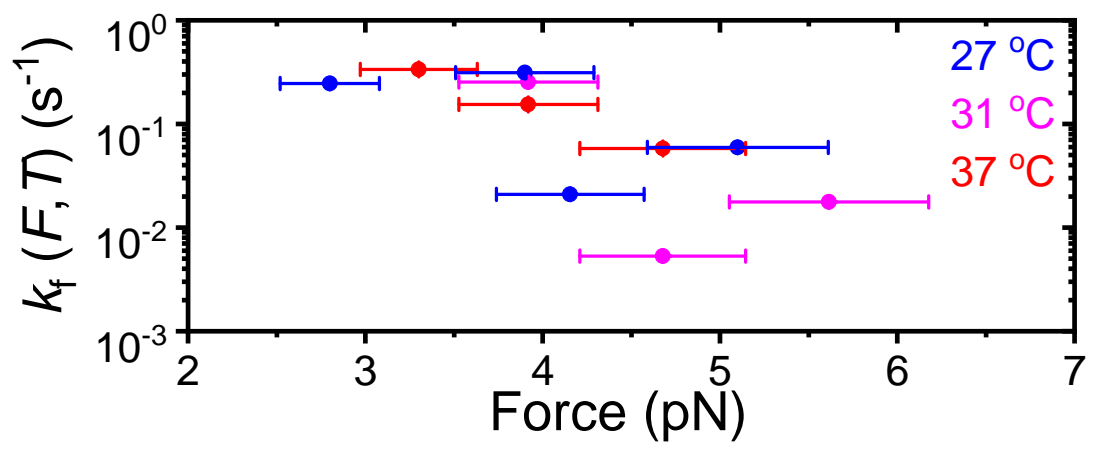

Supplementary Figure S5: The force-dependent refolding rate of I27 at different temperatures.. The solid symbols are the refolding rates of 127 obtained based on experimental measurement at corresponding force and temperature. The x-error bars show the $10 \%$ uncertainty of the force calibration.

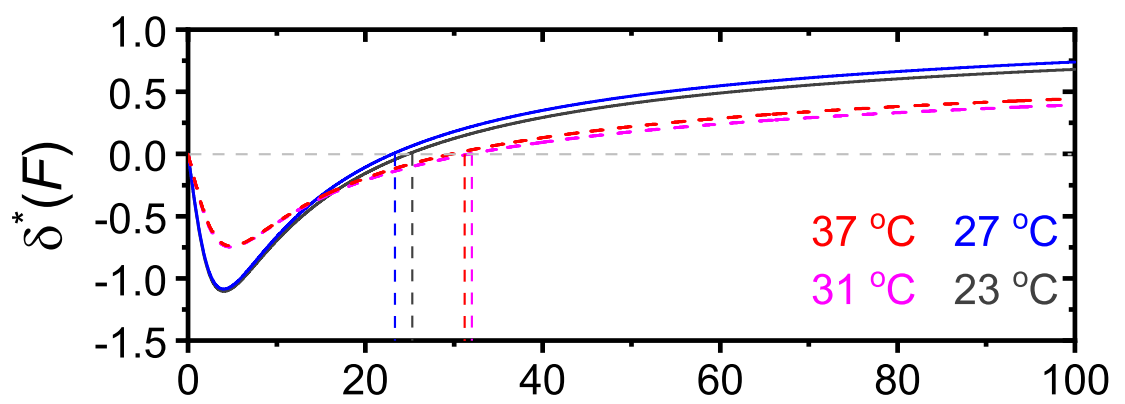

Supplementary Figure S6: The force-dependent transition distance $\left(\delta^{*}(F)\right)$ of I27 unfolding at different temperatures. The four coloured curves show the force-dependent transition distance $\left(\delta^{*}(F)\right)$ of I27 unfolding at different temperatures. The curves are obtained based on the equation $\delta^{*}(F, T)=x^{*}(F, T)-x_{0}(F, T)$. The corresponding parameters used in the equation, i.e., $b^{*}, b_{0}, N^{*}$ are indicated in Figure 2. The vertical lines indicate the force values at which the $\delta^{*}=0$ (indicated by horizontal lines). 

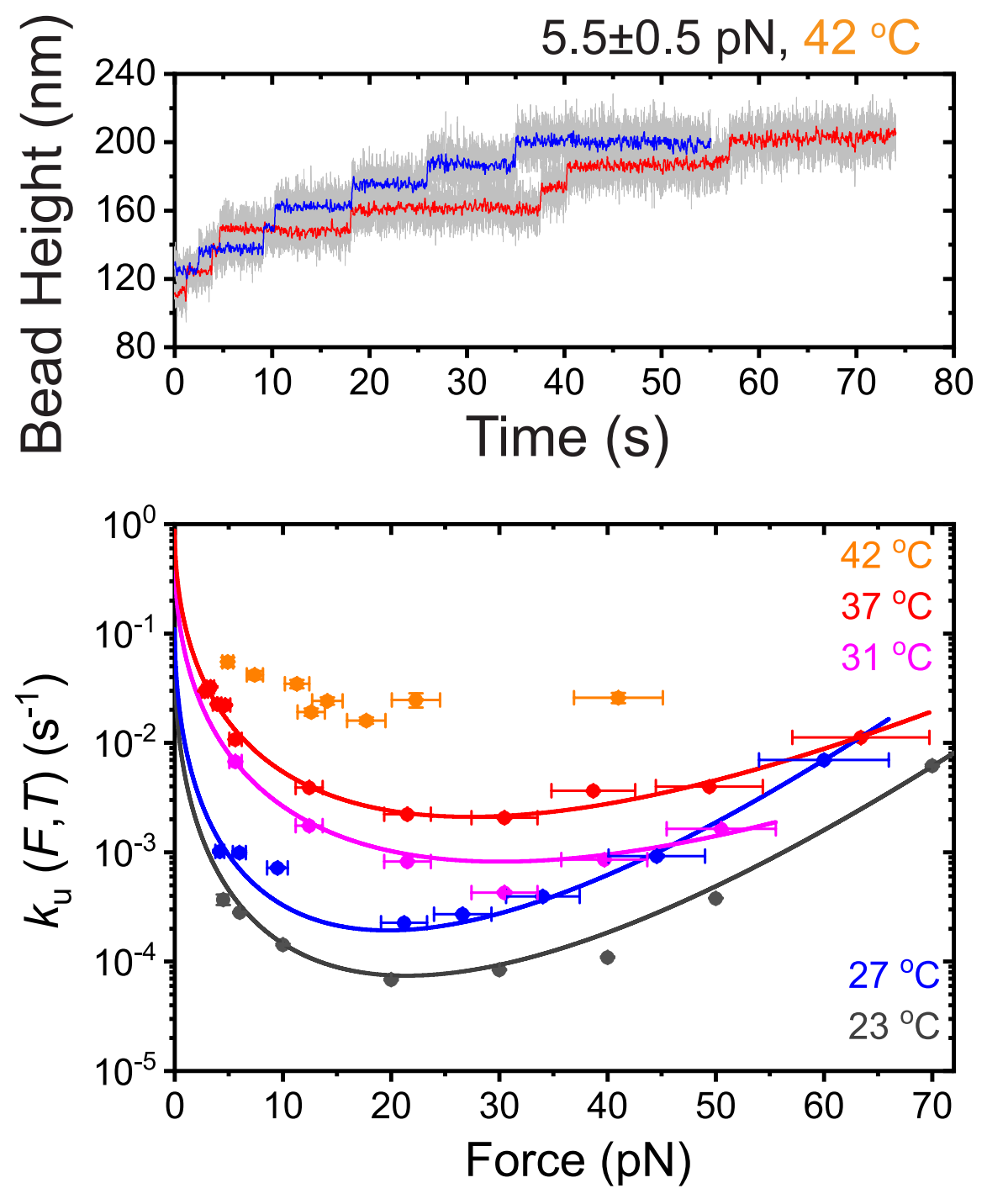

Supplementary Figure S7: The force-dependent unfolding of I27 domain at $42{ }^{\circ} \mathrm{C}$.. Top panel shows two example time-bead height curves of the $\mathrm{I} 27$ domains unfolding at $42^{\circ} \mathrm{C}$ at $5.5 \pm 0.5 \mathrm{pN}$. Here we note that, in the example cases, when the applied force was jumped from a lower force of $\sim 0.5 \mathrm{pN}$ to the target $5.5 \pm 0.5 \mathrm{pN}$ force, one or two of the I27 in the construct have already been staying in the unfolded state, hence only 7 (red) or 6 (blue) unfolding events were observed over the time. Bottom Panel shows the force-dependent unfolding rates of $\mathrm{I} 27$ at different temperatures. The data obtained from $23^{\circ} \mathrm{C}$ to $37^{\circ} \mathrm{C}$ is the same as in Figure 2 in the main text. 


\section{References}

(1) Le, S.; Hu, X.; Yao, M.; Chen, H.; Yu, M.; Xu, X.; Nakazawa, N.; Margadant, F. M.; Sheetz, M. P.; Yan, J. Mechanotransmission and Mechanosensing of Human alphaActinin 1. Cell Rep 2017, 21, 2714-2723.

(2) Cao, Y.; Kuske, R.; Li, H. Direct observation of markovian behavior of the mechanical unfolding of individual proteins. Biophys $J$ 2008, 95, 782-8.

(3) Yuan, G.; Le, S.; Yao, M.; Qian, H.; Zhou, X.; Yan, J.; Chen, H. Elasticity of the Transition State Leading to an Unexpected Mechanical Stabilization of Titin Immunoglobulin Domains. Angew Chem Int Ed Engl 2017, 56, 5490-5493.

(4) Guo, S.; Tang, Q.; Yao, M.; You, H.; Le, S.; Chen, H.; Yan, J. Structural-elastic determination of the force-dependent transition rate of biomolecules. Chem Sci $\mathbf{2 0 1 8}$, 9, 5871-5882.

(5) Rief, M.; Gautel, M.; Oesterhelt, F.; Fernandez, J. M.; Gaub, H. E. Reversible unfolding of individual titin immunoglobulin domains by AFM. Science 1997, 276, 1109-12.

(6) Marszalek, P. E.; Lu, H.; Li, H.; Carrion-Vazquez, M.; Oberhauser, A. F.; Schulten, K.; Fernandez, J. M. Mechanical unfolding intermediates in titin modules. Nature 1999, 402, 100-3.

(7) Williams, P. M.; Fowler, S. B.; Best, R. B.; Toca-Herrera, J. L.; Scott, K. A.; Steward, A.; Clarke, J. Hidden complexity in the mechanical properties of titin. Nature 2003, 422, 446-9.

(8) Guo, S.; Efremov, A. K.; Yan, J. Understanding the catch-bond kinetics of biomolecules on a one-dimensional energy landscape. Communications Chemistry 2019, 2, 30.

(9) Carrion-Vazquez, M.; Marszalek, P. E.; Oberhauser, A. F.; Fernandez, J. M. Atomic 
force microscopy captures length phenotypes in single proteins. Proc Natl Acad Sci U $S A$ 1999, 96, 11288-92.

(10) Improta, S.; Politou, A. S.; Pastore, A. Immunoglobulin-like modules from titin I-band: extensible components of muscle elasticity. Structure 1996, 4, 323-37.

(11) Chen, H.; Yuan, G.; Winardhi, R. S.; Yao, M.; Popa, I.; Fernandez, J. M.; Yan, J. Dynamics of equilibrium folding and unfolding transitions of titin immunoglobulin domain under constant forces. J Am Chem Soc 2015, 137, 3540-6.

(12) Lu, H.; Isralewitz, B.; Krammer, A.; Vogel, V.; Schulten, K. Unfolding of titin immunoglobulin domains by steered molecular dynamics simulation. Biophys $J$ 1998, 75, $662-71$.

(13) Lu, H.; Schulten, K. Steered molecular dynamics simulations of force-induced protein domain unfolding. Proteins 1999, 35, 453-63.

(14) Best, R. B.; Fowler, S. B.; Herrera, J. L. T.; Steward, A.; Paci, E.; Clarke, J. Mechanical unfolding of a titin Ig domain: structure of transition state revealed by combining atomic force microscopy, protein engineering and molecular dynamics simulations. $J$ Mol Biol 2003, 330, 867-77. 